\title{
ОПРЕДЕЛЕНИЕ ФУНКЦИИ НЕОДНОРОДНОГО РАСПРЕДЕЛЕНИЯ И ОДНОРОДНЫХ СОСТАВЛЯЮЩИХ СПЕКТРОВ ПРИМЕСНЫХ МОЛЕКУЛ
}

Известно, что реальные спектры примеси всегда отражают как свойства самой примеси и ее взаимодействия с идеальным кристаллом (в виде однородной составляющей спектров), так и влияние неоднородностей кристалла (эффекты неоднородного уширения спектров). Обычно некоторыми достаточно специализированными методами исследовался один из названных аспектов примесных систем. Например, лазерным возбуждением удовлетворительно снималось неоднородное уширение спектров и исследовался их однородный вид $\left[{ }^{1,2}\right]$ или, наоборот, методом двойного сканирования измерялась функция неоднородного распределения (ФНР) $\left[{ }^{3}\right]$, которая, являясь важной характеристикой существенно неидеальных примесных систем [4,5], отражает наличие и вид дефектов, их плотность и т. д. в кристаллической матрице $\left[{ }^{6}\right]$.

В настоящей статье развита методика определения в достаточно общем случае как ФНР, так и однородного вида спектров поглощения и люминесценции на основе знания спектра вторичного свечения примесной системы путем решения соответствующего интегрального уравнения.

\section{Теория}

Для описания примесной системы будем исходить из модели $\left[{ }^{5}\right]$. Рассмотрим кристалл со статистически распределенными примесными центрами, каждый из которых обладает своими спектрами люминесценции $\varphi$ и поглощения $x$. Спектр возбуждения $\varepsilon$ будем считать пропорциональным спектру поглощения $x$, квантовый выход люминесценции постоянным и одинаковым для всех центров. Предположим, что неоднородный состав центров выражается только в одинаковых для данного центра смещениях спектров люминесценции и поглощения (возбуждения) и что передача энергии между центрами отсутствует. Тогда интенсивность люминесценции на частоте $v_{f}$ при монохроматическом возбуждении на частоте $v_{e}$ равняется

$$
I\left(v_{e}, v_{f}\right)=A \int_{-\infty}^{\infty} x\left(v_{e}-\omega\right) \varphi\left(v_{f}-\omega\right) \varrho(\omega) d \omega,
$$

где $\varrho(\omega)$ - ФНР центров по величине смещения спектров. $A$ можно считать постоянной (не зависящей от $v_{e}$ и $v_{f}$ ) в случае: 1) пренебрежимо слабой частотной зависимости коэффициента пропорциональности между вероятностью перехода и квадратом матричного элемента опе- 
ратора дипольного возмущения $\left.\left[{ }^{7}\right], 2\right)$ слабого поглощения, когда доли поглощенного света складываются линейно (имеется в виду поглощение не только данных центров, но и всей системы), 3) отсутствия или пренебрежимо слабой реабсорбции люминесценции. Если $I\left(v_{e}, v_{f}\right)$ известна из эксперимента, можно рассматривать (1) как интегральное уравнение относительно подлежащих определению функций $\chi, \varphi, \varrho$. $\mathrm{C}$ применением двойного преобразования Фурье по переменным $v_{e}$ и $v_{f}$ уравнение (1) преобразуется в функциональное:

$$
\tilde{I}\left(t_{e}, t_{f}\right)=\tilde{A} \tilde{x}\left(t_{e}\right) \tilde{\varphi}\left(t_{f}\right) \tilde{\varrho}\left(t_{e}+t_{f}\right),
$$

где $\tilde{I}, \tilde{x}, \tilde{\varphi}$ и $\tilde{\varrho}$ - преобразования Фурье от $I, x, \varphi$ и е соответственно. В дальнейшем для удобства будем писать (2) в логарифмах

$$
\ln \tilde{I}\left(t_{e}, t_{f}\right)=\ln A+\ln \tilde{\varkappa}\left(t_{e}\right)+\ln \tilde{\varphi}\left(t_{f}\right)+\ln \tilde{\varrho}\left(t_{e}+t_{f}\right) \text {. }
$$

Образуем теперь выражение

$$
h(t)=\ln I(t, t)-\ln I(0, t)-\ln I(t, 0)+\ln I(0,0) .
$$

Подставляя сюда соответствующие значения $\ln \widetilde{I}\left(t_{e}, t_{f}\right)$ из (3), получаем

$$
h(t)=\ln \tilde{\varrho}(2 t)-2 \ln \tilde{\varrho}(t)-\ln \tilde{\varrho}(0) .
$$

Будем считать, что @ нормирована на единицу, тогда $\tilde{\varrho}(0)=1$ и $\ln \tilde{\varrho}(0)=0$. Из (5) следует

$$
\ln \tilde{\varrho}(2 t)=2 \ln \tilde{\varrho}(t)+h(t)
$$

или

$$
\ln \tilde{\varrho}(t)=2 \ln \tilde{\varrho}\left(\frac{t}{2}\right)+h\left(\frac{t}{2}\right) .
$$

После $n$-кратного применения (7) получаем

$$
\ln \tilde{\varrho}(t)=2^{n} \ln \tilde{\varrho}\left(\frac{t}{2^{n}}\right)+\sum_{k=1}^{n} 2^{k-1} h\left(\frac{t}{2^{k}}\right) .
$$

Если правая часть (8) сходится при $n \rightarrow \infty$, она определяет искомое решение. Не приводя здесь подробного доказательства, отметим только, что она сходится, если $\varrho(\omega)$ при $|\omega| \rightarrow \infty$ имеет лоренцеву асимптотику или убывает быстрее. Тогда

$$
\lim _{n \rightarrow \infty} 2^{n} \ln \tilde{\varrho}\left(\frac{t}{2^{n}}\right)=i \omega_{0} t-\gamma|t|
$$

где $\omega_{0}-$ вещественная постоянная, $\gamma \geqslant 0$. Функция $h(t)$ не определяет величин $\omega_{0}, \gamma$. Неопределенность $\omega_{0}$ связана с инвариантностью уравнения (1) относительно преобразования

$$
\begin{aligned}
& \varrho(\omega) \rightarrow \varrho\left(\omega+\omega_{0}\right), \\
& x\left(v_{e}\right) \rightarrow \chi\left(v_{e}-\omega_{0}\right), \\
& \varphi\left(v_{f}\right) \rightarrow \varphi\left(v_{f}-\omega_{0}\right) .
\end{aligned}
$$


Отметим, что если существует первый момент распределения $\varrho(\omega)$, то он равен $\omega_{0}$.

Определение $\gamma$ требует дополнительного изучения функции $I\left(v_{e}, v_{f}\right)$. $\gamma \neq 0$ только тогда, когда $\varrho(\omega)$ имеет лоренцеву асимптотику или убывает медленнее. Если $\chi$ и $\varphi$ имеют лоренцеву асимптотику или убывают быстрее, значение $\gamma$ дается формулой

$$
\gamma=\left.\frac{1}{2}\left(\frac{\partial_{-}}{\partial t}-\frac{\partial_{+}}{\partial t}\right) \ln \int_{-\infty}^{\infty} I(v, v-\Delta) \mathrm{e}^{i v t} d v\right|_{t=0},
$$

где $\frac{\partial_{+}}{\partial t}, \frac{\partial_{-}}{\partial t} \quad$ - соответствующие односторонние производные, $\Delta-$ произвольная (с единственным ограничением при выборе: $I(v, v-\Delta) \not \equiv$ $\neq 0)$. Окончательно имеем

$$
\ln \tilde{\varrho}(t)=i \omega_{0} t-\gamma|t|+\sum_{k=1}^{\infty} 2^{k-1} h\left(\frac{t}{2^{h}}\right),
$$

где $\omega_{0}$ - произвольная вещественная постоянная, $\gamma$ определяется из (11), и бесконечная сумма сходится, если @ имеет лоренцеву асимптотику или убывает быстрее. Функция $\tilde{\varrho}(t)$ выражается тогда через бесконечное произведение

$$
\tilde{Q}(t)=\mathrm{e}^{i \omega_{0} t-\gamma|t|} \prod_{k=1}^{\infty} f^{2^{k-1}}\left(\frac{t}{2^{k}}\right),
$$

где

$$
f=\exp (h)=\frac{I(0,0) I(t, t)}{I(0, t) I(t, 0)}
$$

Из (2) имеем

$$
\tilde{x}(t)=\frac{T(t, 0)}{A \tilde{\varphi}(0) \tilde{\varrho}(t)}, \quad \tilde{\varphi}(t)=\frac{T(0, t)}{A \tilde{x}(0) \tilde{\varrho}(t)} .
$$

Функции $\varrho, x, \varphi$ можно получить из (13) и (15) с помощью обратного преобразования Фурье. При строгом подходе, когда учитываются ошибки реальных измерений, в этих вычислениях требуется конечно, некоторая регуляризация, так как уравнение (1) относится к некорректным задачам $\left[{ }^{8}\right]$.

Приведем здесь еще некоторые выражения для моментов ФНР $\varrho(\omega)$. Проще всего они выводятся путем дифференцирования выражения (12), так как значения второй и третьей производных от логарифма характеристической функции $\ln \tilde{\varrho}(t)$ в точке $t=0$ определяют соответственно второй и третий нормированные центральные моменты распределения $\varrho(\omega)$. Для второго момента, который характеризует ширину распределения, получаем

$$
\overline{(\omega-\bar{\omega})^{2}}=\overline{\left(v_{e}-\bar{v}_{e}\right)\left(v_{f}-\overline{v_{f}}\right)}=\overline{v_{e} v_{f}}-\bar{v}_{e} \bar{v}_{f},
$$

и для третьего момента, характеризующего асимметрию распределения, - 


$$
\overline{(\omega-\bar{\omega})^{3}}=\frac{1}{2}\left[\overline{\left(v_{e}-\bar{v}_{e}\right)^{2}\left(v_{f}-\bar{v}_{f}\right)}+\overline{\left(v_{e}-\bar{v}_{e}\right)\left(v_{f}-\bar{v}_{f}\right)^{2}}\right] .
$$

Усреднение в правых частях формул (16) и (17) подразумевается с помощью нормированной функции $I\left(v_{e}, v_{f}\right)$. Так, например,

$$
\overline{v_{e} v_{f}}=\iint v_{e} v_{f} I\left(v_{e}, v_{f}\right) d v_{e} d v_{f}
$$

Аналогичные выражения можно получить и для высших моментов. Эти моменты можно вычислить без решения уравнения (1) на основе знания функции $I\left(v_{e}, v_{f}\right)$.

\section{Экспериментальные возможности}

Выясним, какие измерительные данные требуются для вычислений, т. е. какие функции являются оригиналами для $I(0, t), \widetilde{I}(t, 0)$ и $I(t, t)$, входящих в (4). Применяя к последним обратное преобразование Фурье, можно показать, что для $T(0, t)$ оригиналом является спектр люминесценции (при бесконечно широком возбуждении)

$$
I(0, t) \leftrightarrow I_{f}(v)=\int_{-\infty}^{\infty} I\left(v^{\prime}, v\right) d v^{\prime},
$$

для $T(t, 0)$ - спектр возбуждения интегральной люминесценции

$$
I(t, 0) \leftrightarrow I_{e}(v)=\int_{-\infty}^{\infty} I\left(v, v^{\prime}\right) d v^{\prime},
$$

для $T(t, t)$ - функция $F(v)$, которая выражается как сумма (интеграл) результатов всех измерений, при которых сумма частот возбуждения и регистрации равна $v$ :

$$
I(t, t) \leftrightarrow F(v)=\int_{-\infty}^{\infty} I\left(v-v^{\prime}, v^{\prime}\right) d v^{\prime}
$$

Отсюда видно, что выполнения двумерного преобразования Фурье фактически не требуется - сначала следует лишь провести суммирования (18)(20), а потом вычислить нужные одномерные преобразования Фурье. Суммирования (18) и (19) можно в некоторых случаях с необходимой степенью точности провести экспериментально, применяя широкие возбуждение и регистрацию соответственно, для $F(v)$ же, видимо, единственно возможным является численное суммирование, которое требует знания всех значений $I\left(v_{e}, v_{f}\right)$, отличных от нуля. Практическое применение данного метода и осложняет большое количество требуемых данных. Их число

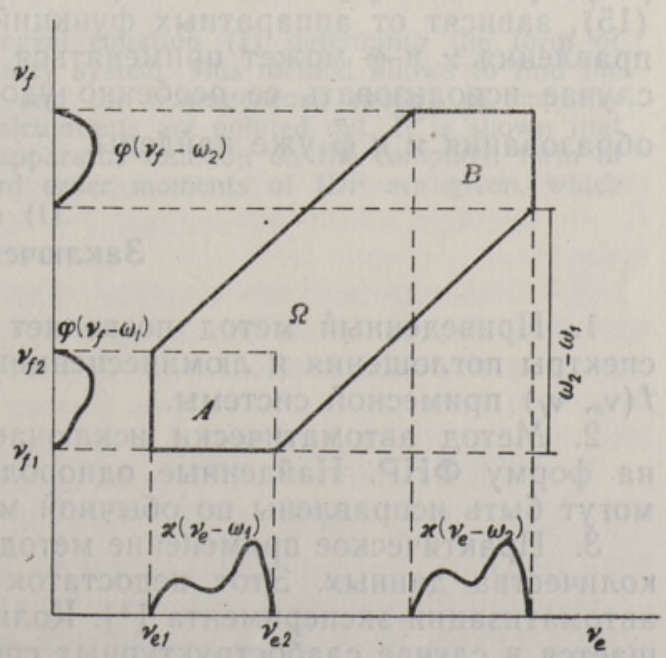

Область $\Omega$ измерения двумерного спектра $I\left(v_{e}, v_{f}\right)$. В прямоугольниках $A$ и $B$ расположены наиболее смещенные однородные спектры. 
можно уменьшить в случае слабоструктурных спектров, так как для их фиксирования нужно меньше точек измерения. Отметим, что прямая методика определения ФНР $\left[{ }^{3}\right]$ здесь не срабатывает. Вторая возможность уменьшения объема эксперимента возникает, когда $\chi, \varphi$ и (или некоторые из них - обычно $x$ и $\varphi$ ) состоят из отделенных друг от друга полос, и нас интересуют только некоторые из функций $x, \varphi$, 0 или отдельные их полосы (рисунок). В этом случае, если $I\left(v_{e}, v_{f}\right)$ на границе некоторой области $\Omega$ плоскости переменных $v_{e}, v_{f}$ равна нулю, то, как видно из структуры уравнения (1), ее значения в этой области определяются только значениями $x\left(v_{e}\right), \varphi\left(v_{f}\right)$ и $\varrho(\omega)$ при $v_{e 1} \leqslant v_{e} \leqslant v_{e 2}$, $v_{f 1} \leqslant v_{f} \leqslant v_{f 2}$ и $\omega_{1} \leqslant \omega \leqslant \omega_{2}$ соответственно. И обратно - из значений $I\left(v_{e}, v_{f}\right)$ в области $\Omega$ можно определить $x, \varphi$ и $\varrho$ в указанных интервалах (приравнивая $I\left(v_{e}, v_{f}\right)$ к нулю вне области $\Omega$ ).

Рассмотрим теперь, как на полученные результаты влияет учет конечной ширины аппаратных функций. В этом случае результат измерений

$$
I^{\prime}\left(v_{e}, v_{f}\right)=\int_{-\infty}^{\infty} \int_{-\infty}^{\infty} g_{e}\left(v_{e}^{\prime}-v_{e}\right) I\left(v_{e}^{\prime}, v_{f}^{\prime}\right) g_{f}\left(v_{f}^{\prime}-v_{f}\right) d v_{e}^{\prime} d v_{f}^{\prime},
$$

где $I\left(v_{e}{ }^{\prime}, v_{f}^{\prime}\right)$ имеет вид $(1), g_{e}$ и $g_{f}$ - аппаратные функции возбуждения и регистрации соответственно. С применением преобразования Фурье (19) принимает вид

$$
\tilde{T}^{\prime}\left(t_{e}, t_{f}\right)=\tilde{g}_{e}\left(-t_{e}\right) \tilde{T}\left(t_{e}, t_{f}\right) \tilde{g}\left(-t_{f}\right) .
$$

Если подставить в (4) вместо $T\left(t_{e}, t_{f}\right)$ значения $T^{\prime}\left(t_{e}, t_{f}\right)$ из $(22)$, то видно, что $h(t)$ не зависит от аппаратных функций. Но тогда от них не зависит и $\mathrm{g}(t)$, вычисляемая по формуле (13). Отметим, что от аппаратных функций не зависят, следовательно, и моменты ФНР (16) и (17). Разрешающая способность метода при определении ФНР будет, разумеется, конечной, но определится она не ширинами аппаратных функций, а статистикой ошибок измерений и применяемым способом регуляризации $\left[{ }^{8}\right]$. В то же время $\tilde{x}$ и $\tilde{\varphi}$, вычисляемые по формулам (15), зависят от аппаратных функций $g_{e}$ и $g_{f}$ соответственно. Для исправления $x$ и $\varphi$ может применяться обычная методика $\left[{ }^{9}\right]$. В данном случае использовать ее особенно удобно, так как нужные Фурье-преобразования $\tilde{x}$ и $\tilde{\varphi}$ уже найдены.

\section{Заключение}

1. Приведенный метод позволяет определять ФНР и однородные спектры поглощения и люминесценции из спектра вторичного свечения $I\left(v_{e}, v_{f}\right)$ примесной системы.

2. Метод автоматически исключает влияние аппаратных функций на форму ФНР. Найденные однородные спектры при необходимости могут быть исправлены по обычной методике.

3. Практическое применение метода связано с измерением большого количества данных. Этот недостаток устраняется внедрением систем автоматизации эксперимента $\left[{ }^{3}\right]$. Количество требуемых данных уменьшается в случае слабоструктурных спектров, а также если нет необходимости в полной информации об $x, \varphi$ и $\varrho$.

Автор благодарен К. Ребане, Р. Авармаа и Т. Тамму за обсуждение настоящей работы и полезные замечания. 


\section{Л И Т Р А Т Р А}

1. S z a b o A., Phys. Rev. Lett., 25, 924 (1970).

2. Персонов Р. И., Альшиц Е. И., Быковская Л. А., Х арламов Б. М., ЖЭТФ, 65, 1825 (1973).

3. Т амм Т. Б., Кикас Я. В., Сирк А. Э., ЖПС, 24, 315 (1976).

4. Ребане К. К., Ав арм а а Р. А., Гороховский А. А., Изв. АН СССР, Сер. физ., 39, 1793 (1975).

5. А в а р м а а Р. А., Изв. АН ЭССР, Физ. Матем., 23, 238 (1974).

6. S to n e ham A. M., Rev. Mod. Phys., 41, 82 (1969).

7. Р еб а н К. К., Элементарная теория колебательной структуры спектров примесных центров кристаллов, М., 1968.

8. Т ур чин В. Ф., Т уровце в а Л. С., Опт. и спектр., 36, 280 (1974).

9. Р а у и а н С. Г., УФН, 66, 475 (1958).
Институт физики
Академии наук Эстонской ССР
Поступила в редакцию $24 /$ XI 1975

\section{J. KIKAS}

\section{LISANDIMOLEKULIDE MITTEHOMOGEENSE JAOTUSFUNKTSIOONI JA HOMOGEENSETE SPEKTRITE MÄÄRAMINE}

Esitatakse lisandisüsteemi sekundaarkiirguse spektri kuju määrava integraalvõrrandi (1) lahendamise meetod. See lubab leida lisandimolekulide mittehomogeense jaolusfunktsiooni (MHJ) ja homogeensed spektrid. Selgitatakse välja arvutusteks vajalikud eksperimentaalandmed. Näidatakse, et meetod välistab aparaadifunktsioonide mõju arvutatud $M H J-i$ kujule. Esitatakse $M H J-i$ teist ja kolmandat järku momentide avaldised, mis on arvutatavad ilma võrrandit (1) lahendamata.

\section{J. KIKAS}

\section{DETERMINATION OF THE INHOMOGENEOUS DISTRIBUTION FUNCTION AND HOMOGENEOUS SPECTRA OF IMPURITY MOLECULES}

A method is given of solving the integral equation (1) determinig the form of the secondary emission spectrum of an impurity system. This method allows to find the inhomogeneous distribution function (IDF) and the homogeneous spectra of impurity molecules. Experimental data required for calculations are pointed out. It is shown that the method eliminates the influence of the apparatus function on the computed form of IDF. The formulae for the second and third order moments of IDF are given, which may be calculated without solving equation (1). 